Journal of Environmental and Agricultural Studies

ISSN: $2710-140$

DOI: $10.32996 /$ jeas

Journal Homepage: www.al-kindipublisher.com/index.php/jeas

JAES

\title{
Hydrogeological Synthesis of Groundwater Resources: Case of the Feija Watershed (South-east of Morocco)
}

\author{
EZ-ZIYANI Aboubakr ${ }^{1}$ 8(D) $\triangle$, TABIT Abdelhalim ${ }^{2}$ (D), ALGOUTI Ahmed ${ }^{3}$ (D), ALGOUTI Abdellah ${ }^{4}$, \\ FARAH Abdelouhed ${ }^{5} \mathbf{8}$ (D) GOULMA Mariam ${ }^{6}$ and ELBADAOUI Kamal $\mathbf{8}^{\mathbf{8}}$ (D) \\ ${ }^{1234567}$ University of Cadi Ayyad, Faculty of Sciences Semlalia, Department of Geology, Geoscience Geotourism Natural Hazards \\ and Remote Sensing Laboratory (2 GRNT), BP 2390, 40000, Marrakech, Morocco
}

$\triangle$ Corresponding Author: EZ-ZIYANI Aboubakr, E-mail: Aboubakrezziyani@gmail.com

\begin{tabular}{|c|c|}
\hline ARTICLE INFORMATION & ABSTRACT \\
\hline Received: March 08, 2021 & \multirow{7}{*}{$\begin{array}{l}\text { The Feija watershed is located in southeast Morocco. It is the main tributary of the } \\
\text { middle Draa valley. It covers an area of } 2089.3 \mathrm{~km}^{2} \text {. The Feija watershed dominated by } \\
\text { an arid climate. The economic development in the Feija basin is essentially based on } \\
\text { agriculture, which requires pumping a considerable amount of groundwater resources. } \\
\text { These waters are being overexploited because of the fast growth in the agricultural } \\
\text { sector and the influence of adverse climatic conditions. The present study concerns a } \\
\text { hydrogeological synthesis of the Feija groundwater, in particular the influence of } \\
\text { topographical, climatic, hydrological and geological factors that interact to create a } \\
\text { complex system with uncontrolled agricultural consumption. To this effect, and to } \\
\text { determine the recharge and discharge rates of the groundwater, the contribution of } \\
\text { remote sensing and GIS presents a major role in assessing and monitoring the } \\
\text { condition of the groundwater and faced the water stress characteristic of this study } \\
\text { area. }\end{array}$} \\
\hline Accepted: May 29, 2021 & \\
\hline Volume: 2 & \\
\hline Issue: 1 & \\
\hline DOI: 10.32996/jeas.2021.2.1.9 & \\
\hline KEYWORDS & \\
\hline $\begin{array}{l}\text { Feija watershed, Middle Draa } \\
\text { valley, Groundwater resources, } \\
\text { Agriculture. }\end{array}$ & \\
\hline
\end{tabular}

\section{Introduction}

Dans les zones arides, la gestion des ressources en eau est un défi, dont la difficulté s'accroît progressivement en raison, d'une part, de l'influence des changements climatiques, et d'autre part, de l'évolution démographique de la population et de leurs besoins en eau. Étant donné que l'eau est un facteur limitant pour le développement, surtout dans les régions arides, le Maroc est un pays plus vulnérables aux effets du changement climatique (Boko et al. 2007), ainsi qu'il présente 93\% de sa superficie qui soumit au climat aride et semi-aride, le bassin de Draa représente les conditions climatiques arides extrême (Chamayou, 1966 ; Hübener et al. 2005). La moyenne vallée de Draa est un exemple typique d'un bassin hydrographique aride d'où les ressources en eau de surface et souterraine sont interconnectées hydrauliquement et représente une salinité élevée (Heidecke, 2009 ; El Mandour, 2007). En fait la région de Zagora parmi les zones les plus arides du royaume qui se caractérise par la rareté en matière de l'eau, ce problème de l'eau est toujours d'actualité dans la région, en effet l'alimentation en eau potable se fait d'une partie par la station de déminéralisation des eaux de la nappe Ennebch et d'autre partie provenant de la nappe phréatique de la Feija, ce dernier soumis à une surexploitation agricole qui va conduit à l'épuisement accéléré des réserves de cette nappe. Pour évaluer les volumes soutirés par pompage, on peut extraire les surfaces cultivées à l'aide de la télédétection qui va servir à la détermination des volumes prélevés à la base des besoins en eau des différents types de cultures.

\section{Cadre général de la région d'étude}

La région d'étude est située à l'extrémité Sud-Est du Royaume Marocain dans la province de Zagora, sur le bassin du Drâa qui s'étale sur une longueur d'environ $1200 \mathrm{Km}$, entre les sommets du Haut Atlas qui culmine à plus de $4000 \mathrm{~m}$ d'altitude et l'embouchure de l'oued dans l'océan Atlantique au niveau de la région de Tan-Tan.

Le moyen Drâa s'étendu sur une superficie de 13483 km² drainé principalement par l'oued Drâa alimenté par des affluents le long de sa trajet à partir de barrage Mansour Eddahbi en amont et la palmeraie de M'hamid en aval. Sur la rive droite de la vallée

\section{K C AL-KINDI CENTER \\ $R$ DFOR RESEARCH AND DEVELOPMENT}

Your gateway to world-class research

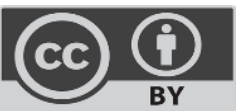

Published by Al-Kindi Center for Research and Development, London, United Kingdom. Copyright (c) the author(s). This open access article is distributed under a Creative Commons Attribution (CC-BY) 4.0 license 
moyenne Drâa et à l'ouest de la palmeraie de Fezouata, la plaine de l'oued Feija s'étend sur une superficie de 934 km², de Foum Zguid à l'Ouest jusqu'au Foum Zagora au Nord et Foum Takkat au Sud. Le grand reg du bassin Feija donnant déjà la mesure des grands espaces du désert, et limité au Sud par le Jbel Bani c'est la dernière chaîne montagneuse qui sépare les chaînes anti atlasique du domaine Saharienne.

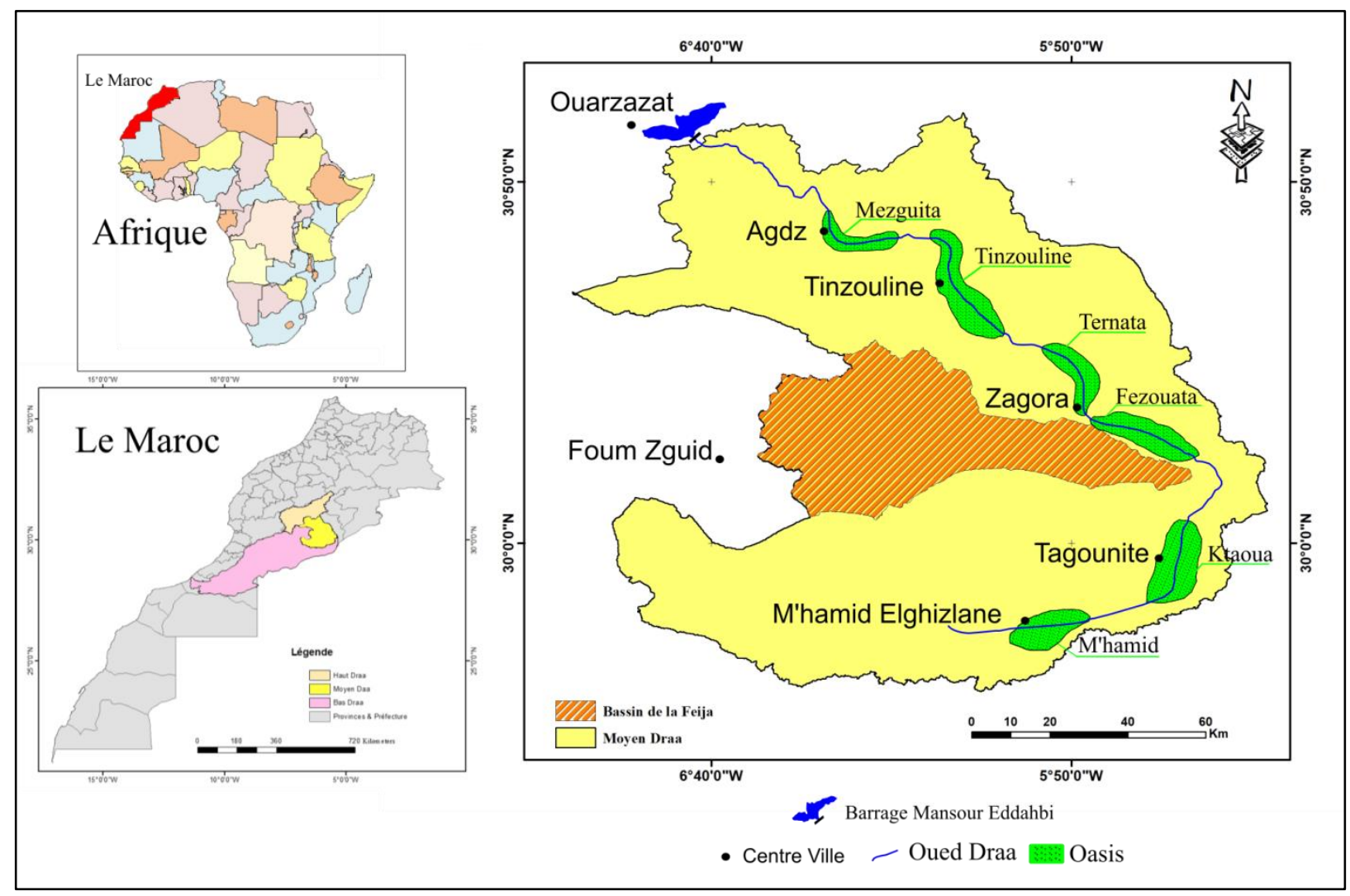

Figure 1. Localisation géographique de la zone d'étude.

\section{Méthodologie}

\subsection{Cartes topographiques}

À partir de la mosaïque des cartes topographique 1/100000 de Foum Zguid, El Gloa et celle de Zagora on peut localiser le bassin versant de la Feija et extraire de l'information topographique et hydrographique caractéristique de la zone d'étude, ainsi les localités et le réseau routier.

\subsection{Cartes géologiques}

Le bassin versant de l'oued Feija est couvert par la carte géologique de Zagora- code du dra- Hamada du dra 1/200000 qui couvre presque la totalité du bassin et la carte géologique de l'anti-atlas central et de la zone synclinale de Ouarzazate 1/200000 qui couvre une petite partie à l'ouest du bassin.

\subsection{Apport de la Télédétection}

L'application de la télédétection facilite le suivi et la surveillance à long terme de la surface terrestre, en se basent sur l'analyse du rayonnement électromagnétique qu'il s'agit d'une énergie transportée dans l'espace sous forme d'ondes (Pouchin, 2001), à partir de l'analyse de l'image satellitaire de la zone d'étude on peut ressortir la dynamique d'occupation du sol. Pour évaluer les volumes d'eau prélevée de la nappe de la Feija, la télédétection permet de créer une base d'approche globale, dont les résultats sont significatifs et pratique. 

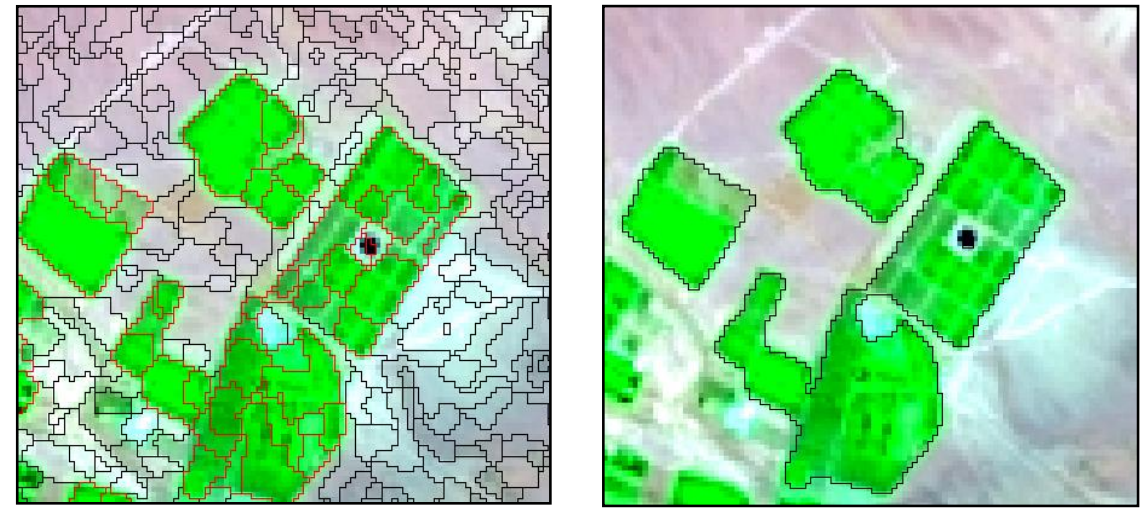

Figure 2. Segmentation de l'image satellitaire et extraction des superficies irriguées.

\subsection{Données des forages et des puits}

La plaine de la Feija est traversée par plusieurs forages à différents but (exploitation, reconnaissance ...), ces forages disposant des fiches IRE, les données collectées concernent les épaisseurs et la description lithologique des différents horizons traversés et aussi le niveau piézométrique.

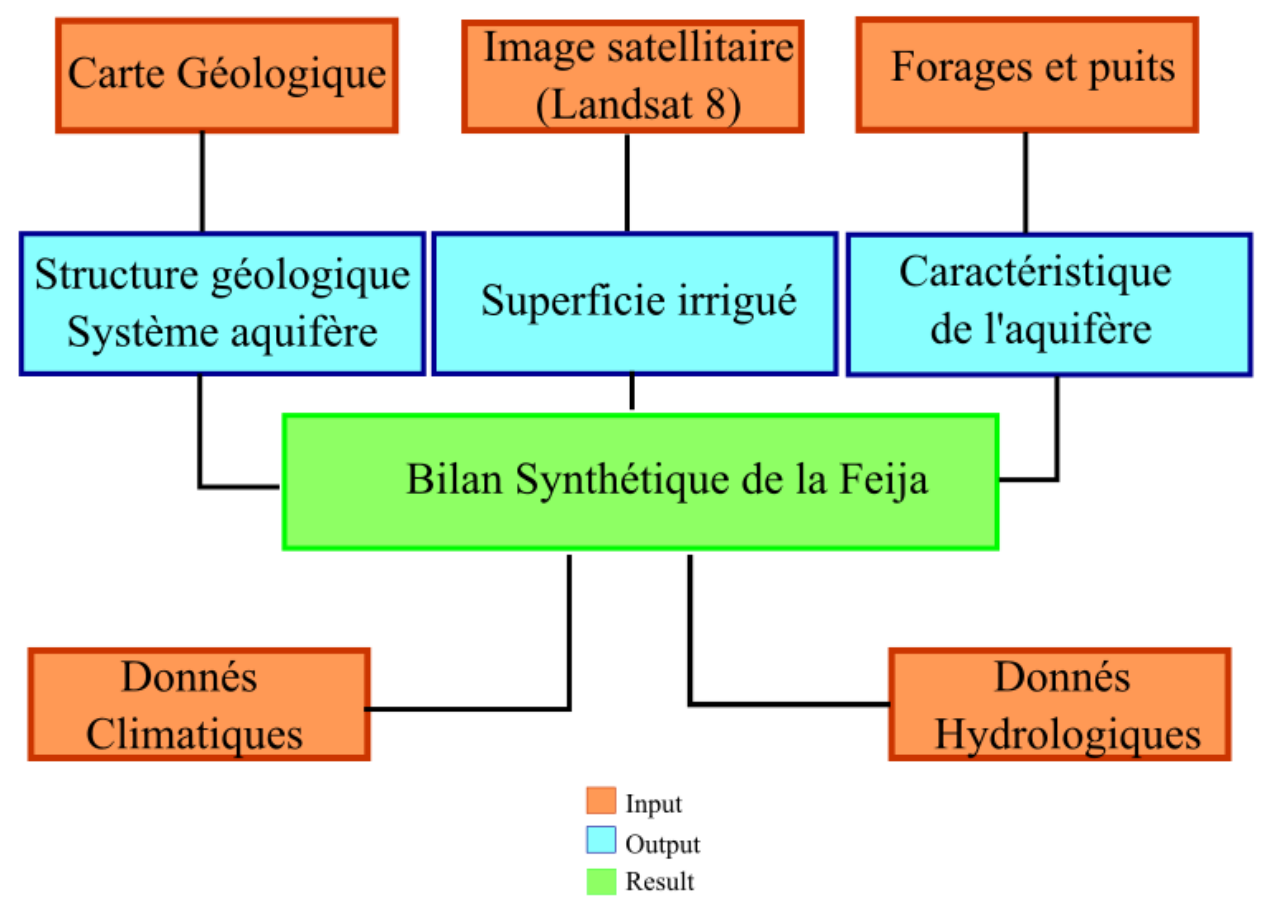

Figure 3. Méthodologie de travail.

\section{Résultats et Discussion}

\subsection{Précipitations}

Le régime annuel des précipitations est caractérisé par quatre saisons de durée inégale, l'été constitue la période quasi-dominant, car il peut durer de quatre à cinq mois inclus les mois d'avril et août. L'évolution moyenne annuelle dans les stations de Zagora et Foum Zguid offrent les valeurs représentées dans le tableau 1.

Il apparaitre clairement que l'évolution est quasi-symétrique avec un régime pluvial très irrégulier dont laquelle se succèdent des périodes humides (1955, 1966, 1968, 1980, 1988, 1990, 2007), et des périodes sèches en dessous de la moyenne (1934, 1937, 1938, 1960, 1961, 1976, 1983, 1999). 
Tableau 1. Pluviométrie moyenne annuelle.

\begin{tabular}{ccc}
\hline Station & Altitude & $\begin{array}{c}\text { Moyenne } \\
\text { annuelle }(\mathrm{mm})\end{array}$ \\
\hline Zagora & 707 & 67,59 \\
Foum Zguid & 650 & 83,64 \\
\hline
\end{tabular}

- La carte des isohyètes est établie sur la base des pluies annuelles corrigées, elle est basée sur la moyenne interannuelle des pluies calculée sur toute la période de (1932-2010). La méthode des isohyètes reposes sur une interpolation de la surface définie par les précipitations mesurées à chaque site pluviométrique.

- La méthode des polygones de Thiessen fait appel à des propriétés géométriques pour déterminer la région d'influence, et donc le poids de chaque station.

Selon la méthode de Thiessen et des isohyètes la pluviométrie moyenne du bassin versant de la Feija et de l'ordre de 75,70mm.

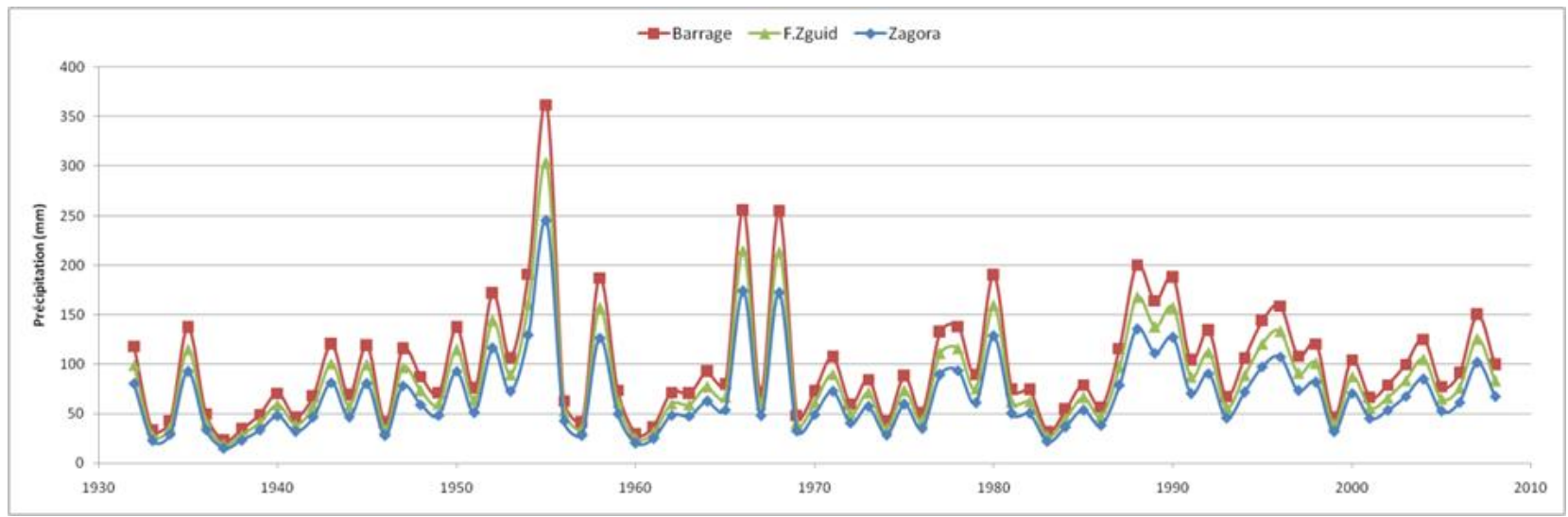

Figure 4. Hauteurs des précipitations moyennes annuelles à Ouarzazate, Zagora et Foum Zguid.

\subsection{Température}

On peut conclure à partir des données des stations de Zagora et Tagounite que le climat dans la région est saharien, les étés sont très chauds avec une température moyenne maximale de $43.84^{\circ} \mathrm{C}$, et les hivers sont froids, avec une température moyenne minimale de $2.05^{\circ} \mathrm{C}$.

\subsection{Evaporation}

Les valeurs moyennes de l'évaporation mensuelle au niveau de la station de Zagora mesurée au bac Colorado, le pouvoir évaporant du bassin est très fort, les valeurs maximales enregistrées concernent les mois de Juillet et Août, qui correspond, d'après les mesures de températures moyennes mensuelles aux mois les plus chauds.

Les zones de pluviométrie de l'ordre de 50 à $80 \mathrm{~mm}$, l'évaporation enlève $100 \%$ des eaux tombée, seul les crues ou les épandages naturelle ou artificielle peut alimenter réellement la nappe (Robaux, 1952). En raison de la faible quantité de précipitation en comparaison avec la quantité évaporée, l'infiltration directe des eaux de pluie est considéré donc négligeable.

\subsection{Contexte Hydrologique}

Le bassin versant de l'oued Feija est caractérisé par une superficie très important avec une topographie assez variable, avec des reliefs anti-atlasique d'altitude compris entre $1717 \mathrm{~m}$ et $609 \mathrm{~m}$, la constatation de terrain et l'analyse du réseau hydrographique ont permet de dresser une vision claire sur les principaux cours d'eau caractérisant le secteur d'étude. Le bassin de la Feija est alimenté par une multitude d'affluents débouchant des reliefs de l'Anti-Atlas à substratum schisteux qui sont Bou Tious, Lbtana, Bou Trid, Es Sadra et Gloa. Le bassin est orienté Est-Ouest, en effet, l'écoulement globale est de direction Est-Oust vers l'oued moyen Draa.

En absence des mesures hydrométriques directes au niveau du bassin versant, les apports seront estimés par deux méthodes: Modélisation Pluie-Débit dans le cadre du Plan Directeur d'Aménagement Intégré des Ressources en Eau (PDAIRE) (2011), d'où l'évaluation des apports dans le moyen Draa (rive droit et rive gauche) sont calculés à l'aide de la série pluviométrique mensuelle de la station de Zagora (1931-2003) et l'évaporation potentielle mensuelle de la station d'Assaka (1931-2003) (ABHSMD, 2013) . 
Méthode basé sur les paramètres hydrologiques : ces paramètres hydrologiques sont les débits spécifiques et les coefficients de ruissellement. A la base du coefficient de ruissellement du bassin de Zagora on peut estimer les apports naturels du bassin de la Feija par une simple multiplication de superficie, précipitation et coefficient de ruissellement (tableau 2).Le débit moyen ruisselé au niveau du bassin versant de la Feija est estimé de 10Mm3/an. On considère que l'infiltration des eaux de crue est de $10 \%$ du volume total ruisselé, le volume d'eau infiltré et de l'ordre de $1 \mathrm{Mm}^{3} / \mathrm{an}$.

Tableau 2. Les apports annuels au niveau du bassin versant de la Feija.

\begin{tabular}{|c|c|c|c|c|}
\hline Bassin & Superficie (Km2) & Précipitation (mm) & $\begin{array}{c}\text { Coefficient de } \\
\text { ruissèlement }(\%)\end{array}$ & $\begin{array}{c}\text { Apport du bassin } \\
(\mathrm{Mm} 3)\end{array}$ \\
\hline Feija & 2089,31 & 75,7 & 6,3 & 9,97 \\
\hline
\end{tabular}

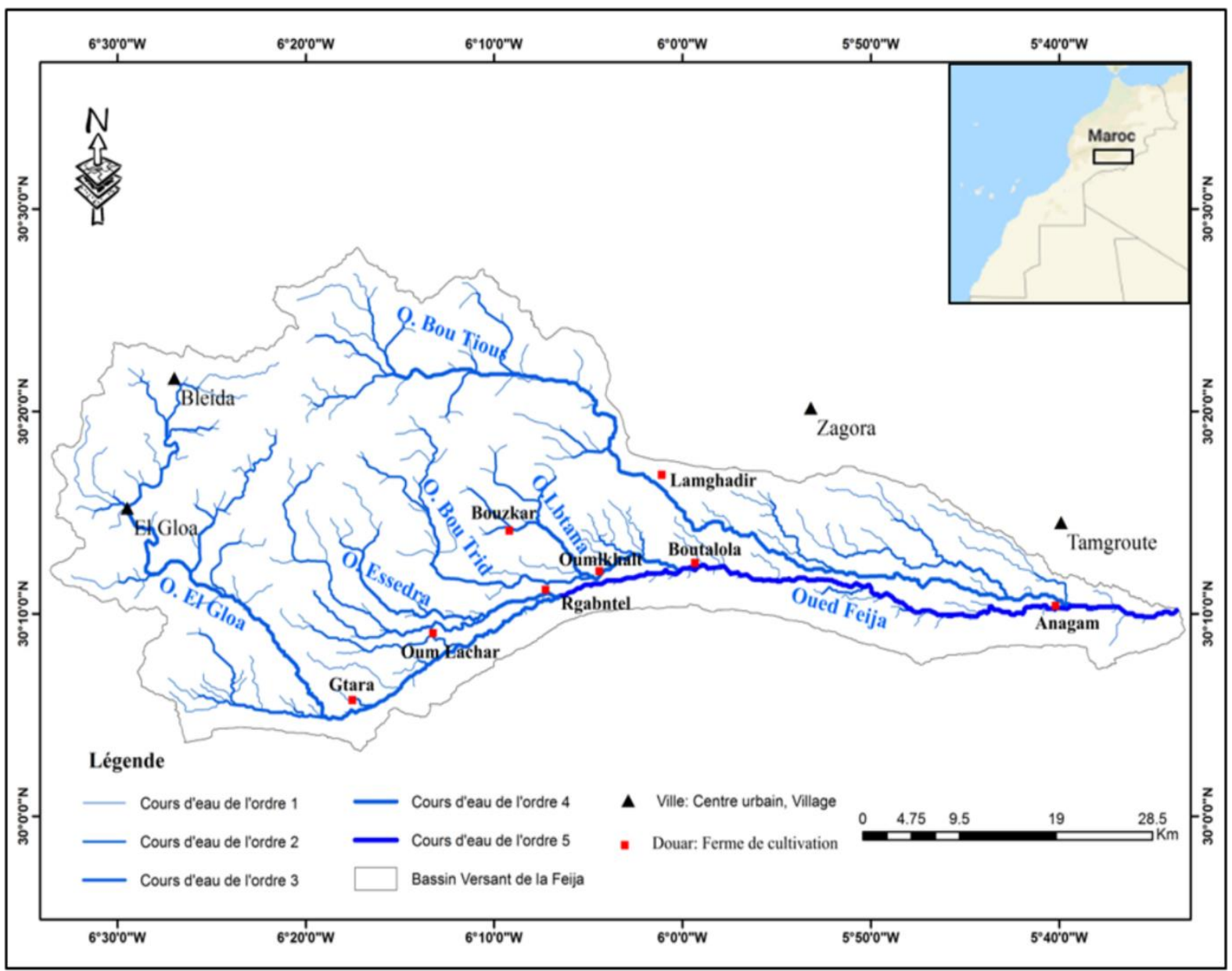

Figure 5. Carte du réseau hydrographique des principaux affluents de l'oued Feija.

\subsection{Géologie du Bassin Feija}

L'étude de la géologie du bassin Feija mettre en évidence des formations géologiques très étendues allant du précambrien jusqu'au quaternaire Les terrains précambriens affleurent en multiples massifs et boutonnières constitués essentiellement par des terrains granitisés, magmatisés et métamorphisés. Sur lesquelles, repose en discordance angulaire (Cherkaoui et al, 2007), une couverture sédimentaire paléozoïque, ce dernier elle-même recouverte par des terrains quaternaires. 


\subsubsection{Socle Protérozoïques}

Litho-stratigraphiquement ce sont des formations granitisé et métamorphisé, ces terrains précambriens sont classés en plusieurs unités successives, la subdivision adoptée par (Choubert, 1952, 1963) est la suivante : PI (Archéen et Paléoprotérozoïque), PII (Néoprotérozoïque), PIII (Néoprotérozoïque terminal) sur laquelle s'ajoutait l'Adoudounien ou Infracambrien.

Le dépôt de l'Adoudounien après les Conglomérats de base (Buggish et Flügel, 1988 ; Algouti et al., 2001), il comprend aux Calcaires inférieurs, la série Lie de vin et les Calcaires supérieurs.

La série lie-de-vin constituée par des schistes et des grès violacés intercalé entre les calcaires inférieurs et calcaires supérieurs, ce dernier contiennent des faunes cambriennes (Piqué et al, 2006) donc appartient au moins en partie au Cambrien.

\subsubsection{Couverture paléozoïque}

La couverture paléozoïque à structure monoclinique dominée par des puissantes séries schisteuses de faible compétence intercalée par des niveaux gréseuses et quartzitiques assez compétents. Ceci explique la morphologie caractéristique de la zone d'étude sous la forme de succession de cuestas.

Après les formations géologiques du Précambrien, la première transgression marine a eu lieu au cours du Cambrien inférieur (Choubert et Marçais, 1952). Entre les calcaires adoudouniens et les grès-quartzites de la fin du Cambrien moyen, les schistes peu résistants à l'érosion déterminent un creux, connu sous le nom de Feija interne. Le cambrien inférieur est constitué par une série de schistes argileux se termine par le niveau marqueur des Grès roses. Le sommet des Grès roses marque la fin du Cambrien inférieur.

Le Cambrien moyen est constitué par les formations du groupe des Feijas internes (Choubert, 1943). Lithologiquement le cambrien moyen débute par des schistes et se poursuit par une puissante série de grès et quartzites dont l'épaisseur peut atteindre $500 \mathrm{~m}$. Les niveaux argilo-gréseuses sommitale du groupe Tabanit contient des débris de trilobites Bailiella sp; avec un pendage de $10^{\circ}$ vers la plaine de la Feija.

L'érosion des terraines de Ordovicien détermine la topographie appalachienne de I'Anti Atlas, d'après (Choubert, 1963) en distingue quatre groupes, Groupe des Feija externe, Groupe du Premier Bani, Groupe de Ktaoua et le Groupe du deuxième Bani. Le groupe des Feijas externes occupent le sous-sol de la grande plaine de la Feija de Zagora et constitue le substratum de la dépression comblée par des alluvions Plio-Quaternaire. Située entre les reliefs gréseuses du cambrien moyen et du premier Bani de Ordovicien moyen, selon (Destombes, 1960) on distingue deux série schisteuses, celle de Fezzouata avec une couleur noir en état franc surmontée par celle de Tachilla de couleur gris-bleu avec une intercalation de faible épaisseur gréso-quartzitique de Zini, l'épaisseur de l'ensemble est avoisine de $1200 \mathrm{~m}$ dont $150 \mathrm{~m}$ pour les schistes supérieur de Tachilla.

Le Jbel Bani composé essentiellement par des matériaux détritiques et comporte deux séries gréseuses, le premier et le deuxième Bani entrecoupées par les schistes dite schiste de Ktaoua, cette succession lithologique atteint environ $600 \mathrm{~m}$ dont $300 \mathrm{~m}$ appartient au premier Bani dans la région d'étude.

\subsubsection{Couverture Plio-Quaternaire}

La couverture plio-quaternaire représenté dans la zone d'étude par des formations alluvionnaires, sableux et limoneux qui représente les secteurs irrigués les plus fertiles de la région.

Une érosion profonde qui exerce sur les éléments lithologiques moins résistants (schiste argileux), le bassin de la Feija est établis en grande partie sur ces terrains et obtenue par la mise en place des terrains plio-quaternaire comble les dépressions. La déformation importante est au cours de la phase tectonique hercynienne d'où la prédominance d'une vaste ondulation des terrains affectés qui évoluent en succession de larges structures synclinales et anticlinale qui se présente sous forme des crêtes parallèles en roches dures de grès-quartzites, entre lesquelles se trouvent des dépressions en roches tendres de schistes (relief appalachien), dans le cadre régional on distingue trois directions hercyniennes principales, se sont superposées sur le plissement précambrien sans se confondre avec celle-ci. La direction dominant NE-SW sensiblement parallèle à la direction globale de la chaine HautAtlasique (Farah et al ,2021), la deuxième direction NW-SE suivie la direction de la boutonnière de Bou Azzer-El Graara et dernièrement la direction mesétienne quasi méridienne (Choubert, 1952), ces familles de fractures affect les formations gréseuse verdâtre sommital du cambrien moyen représentent les mêmes directions. 


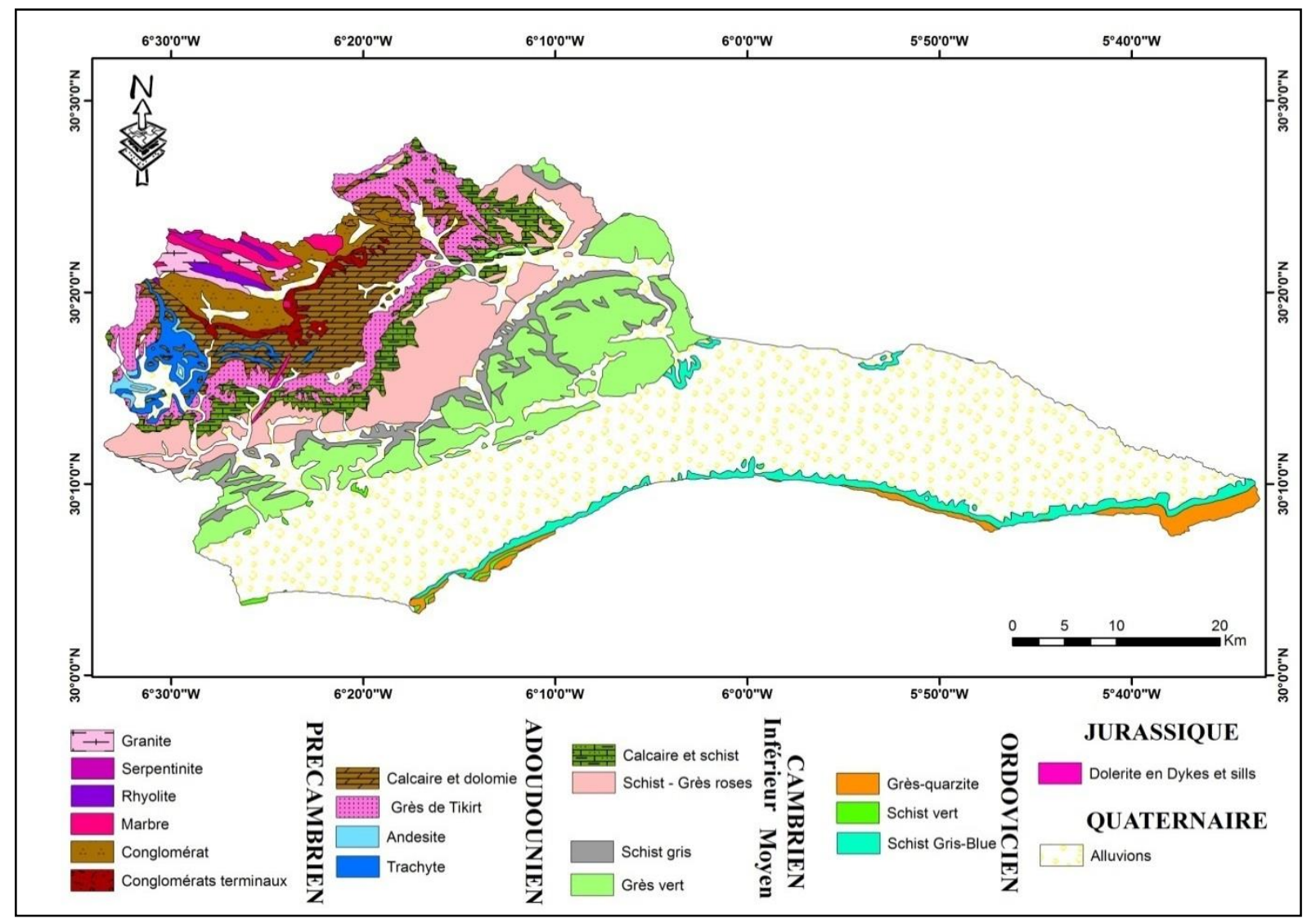

Figure 6. Carte géologique de la zone d'étude (extraite de la carte lithologique du projet IMPETUS Atlas du Maroc 2000-2007; et des cartes géologiques de Zagora- code du dra- Hamada du dra et Anti-atlas central et de la zone synclinale d'Ouarzazate 1/200000).

\subsection{Cadre hydrogéologique}

L'inventaire des études géologique a permis d'identifier la nappe alluviale de la plaine de la Feija, une alimentation continue de la nappe provient de la chaine anti-atlasique de Bou Azzer-El Graara. En principe la circulation de la nappe s'effectue au niveau des terrains alluvionnaires d'âge plio-quaternaire qui se déposent en discontinuité angulaire sur les schistes de l'ordovicien, d'après les observations et les mesures de terrain, les formations grés-quartzitique d'âge cambrien présente un pendage de $10^{\circ}$ vers le sud (vers la plaine de la Feija).

La plaine de la Feija couvre en grande partie des terrains généralement schisto-argileux de l'ordovicien inférieur et moyen audessous des matériaux détritiques quaternaire, ces formations géologiques est caractérisé par une variation spatial des terrains perméables et imperméables, on peut caractériser hydro-géologiquement les formations étudiées, par conséquence générer la carte des systèmes aquifères (Figure 7).

Les eaux souterraines de la nappe Feija sont généralement de moyenne à faible minéralisation par identification de deux principaux faciès (faciès bicarbonaté calcique et le faciès sulfaté calcique) (ABHSMD, 2013). La qualité médiocre est limitée aux zones vulnérables au phénomène d'assèchement, la conductivité électrique est généralement comprise entre $530 \mu \mathrm{s} / \mathrm{cm}$ et $1995 \mu \mathrm{s} / \mathrm{cm}$. Ces mesures représentent la bonne qualité des eaux, en revanche sous l'effet de la pression croissante sur les eaux souterraines, les niveaux actuels des eaux sont plus bas que ceux de l'état naturel. Autrement dit la surexploitation influence la dégradation de quantité et aussi de la qualité, c'est ce qu'est engendré par l'augmentation de minéralisation par lessivage des terrains responsable de la salinité de certain zone, c'est le cas de la nappe Ennebch a l'entourage des périmètres irrigué de nappe Feija. 


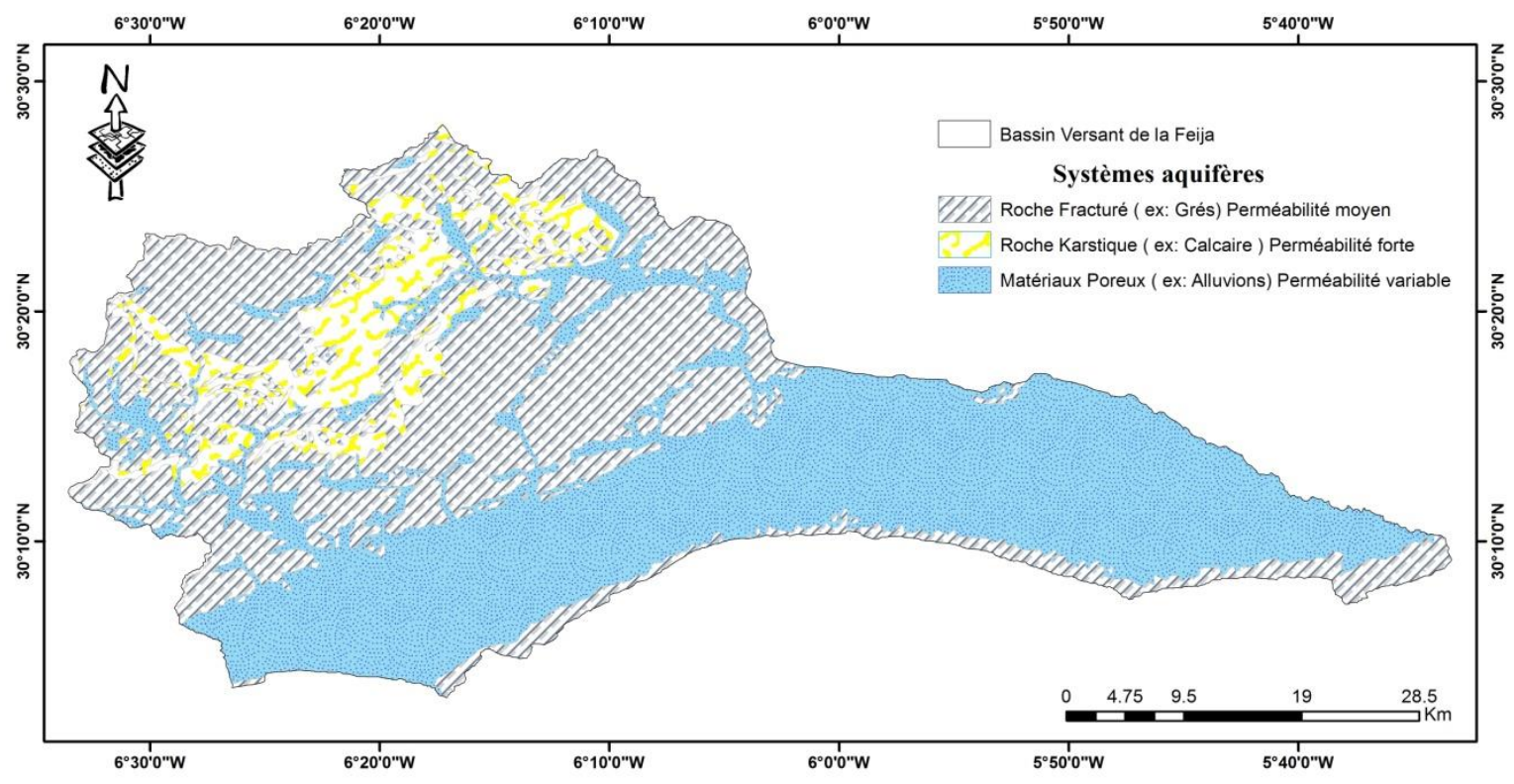

Figure 7. Carte des systèmes aquifères du bassin versant de l'oued Feija.

Une coupe hydrogéologique interprétative permettant de conceptualiser le système aquifère de la nappe Feija, la coupe A-B orienté Nord-Sud permet de se rendre compte de la structure générale des formations géologique qui occupe le sous-sol de la plaine de la Feija (grés-quartzite, schiste argileux et les dépôts alluviales), et apports les renseignements nécessaire sur la dynamique hydrogéologique de la zone d'étude en relation avec la perméabilité et l'inclinaison des couches géologiques. En effet les terrains grès-quartzitiques cambrien fortement fracturées et altérés favorisent l'infiltration des eaux de ruissellement, souterrainement ces eaux collectées ce rassemble et converge vers les petits cluses principalement au niveau de Oued Bou Tious et Oued Lbtana et pénétrant les barres quartzitiques et laisse circuler facilement l'eau et donc constitue un formidable réservoir de la plaine de la Feija a des niveaux assez profonds. Les schistes argileux peu perméable occupe le sous-sol de la plaine de la Feija et constitue à certain endroit des freins à l'écoulement souterrain d'où la variabilité spatiale des ressources en eau souterrains. L'oued Feija suit l'axe de la cuvette à substratum schisteux, il est alimenté par une multitude d'affluents débouchant de l'Anti-Atlas, au niveau de la plaine alluviale de la Feija, Le comblement quaternaire couvre l'ensemble des terrains paléozoïque, la nappe plioquaternaire est constitué par des dépôts quaternaires poreux, parmi ses caractéristiques la faible pente et la profondeur important, qui attient a certaine endroit plus de 100m (zones de cône de déjection). Ce qui permet de drainer l'ensemble des eaux du bassin.

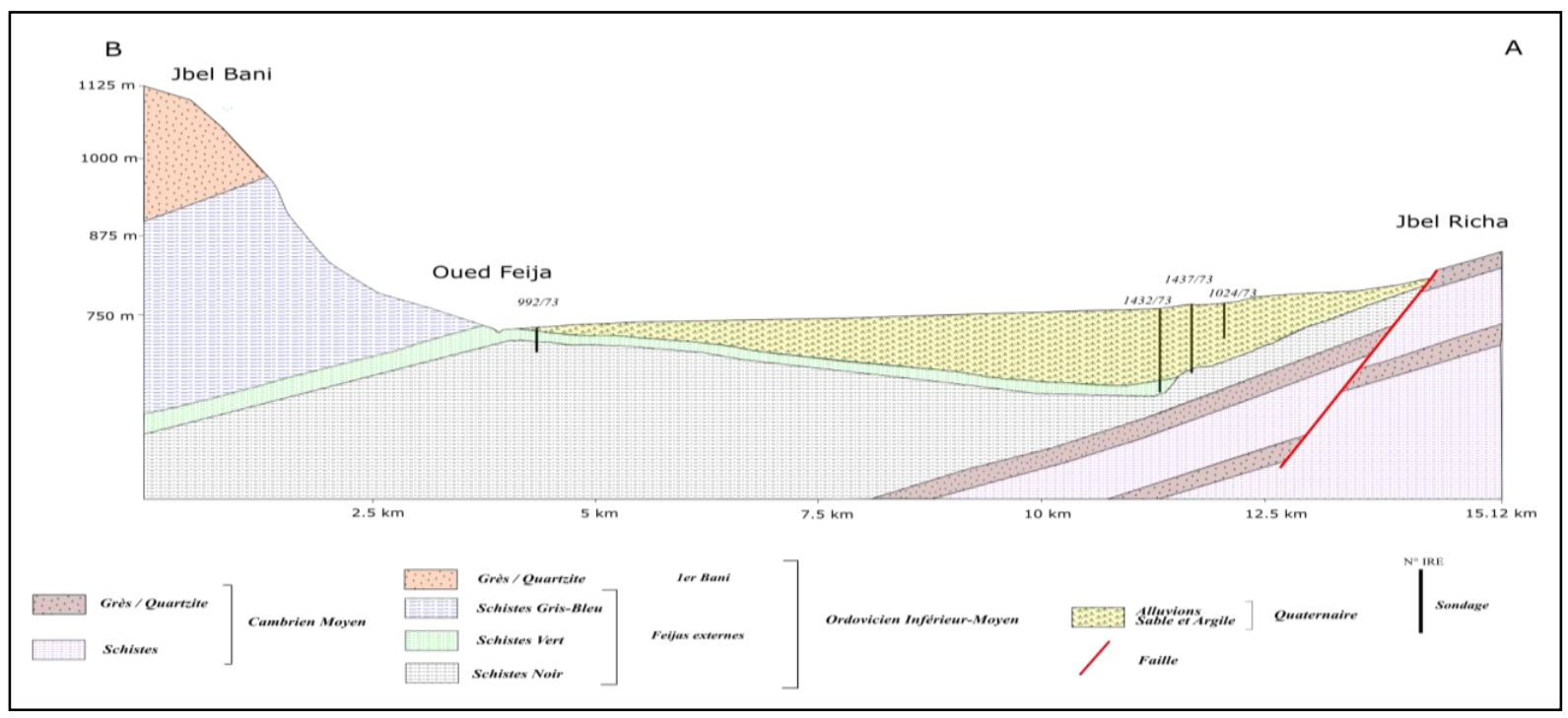

Figure 8. Coupe Hydrogéologique à travers la plaine de la Feija. 
Selon les résultats de l'enquête, la pastèque occupe la majorité des superficies irriguées avec $71.64 \%$. À partir des différentes valeurs théoriques des consommations de chaque type de culture on peut calculer le volume d'eau prélevé par pompage.

Tableau 3. Calcule du volume d'eau prélevé par pompage.

\begin{tabular}{|c|c|c|c|c|}
\hline Type de culture & $\begin{array}{c}\text { Surface irriguée } \\
(\mathrm{ha})\end{array}$ & $(\%)$ & $\begin{array}{c}\text { Dotation } \\
\left(\mathrm{m}^{3} / \mathrm{ha}\right)\end{array}$ & $\begin{array}{c}\text { Volume prélevé } \\
\left(\mathrm{m}^{3} / \mathrm{an}\right)\end{array}$ \\
\hline Pastèque & 1296,90 & 71,64 & 6500 & 8429855,28 \\
\hline Henné & 149,66 & 8,27 & 16000 & 2394606,62 \\
\hline Palmiers & 131,00 & 7,24 & 9000 & 1178970,44 \\
\hline Luzerne & 97,20 & 5,37 & 8000 & 777580,50 \\
\hline Blé dur & 86,33 & 4,77 & 8000 & 690649,35 \\
\hline Petite pois & 23,67 & 1,31 & 3000 & 70998,22 \\
\hline Pomme de terre & 21,33 & 1,18 & 3000 & 63998,40 \\
\hline Gombo & 1,33 & 0,07 & 8000 & 10666,40 \\
\hline Haricotes & 1,00 & 0,06 & 3000 & 2999,92 \\
\hline Cantaloup & 1,00 & 0,06 & 4500 & 4499,89 \\
\hline Oignions & 0,67 & 0,04 & 3000 & 1999,95 \\
\hline Carotte & 0,33 & 0,02 & 3000 & 999,97 \\
\hline
\end{tabular}

Tableau 4. Bilan synthétique de la nappe de la Feija.

\begin{tabular}{|c|c|c|c|}
\hline \multicolumn{2}{|l|}{ ENTREES (Mm³/an) } & \multicolumn{2}{|c|}{ SORTIES ( $\left.\mathrm{Mm}^{3} / \mathrm{an}\right)$} \\
\hline $\begin{array}{l}\text { Infiltration de la pluie et du } \\
\text { ruissellement dispersé }\end{array}$ & Négligeable & Prélèvements agricoles & 13,62 \\
\hline $\begin{array}{c}\text { Alimentation à partir des } \\
\text { bordures }\end{array}$ & 8,89 & $\begin{array}{l}\text { Alimentation en Eau } \\
\text { Potable (AEP) }\end{array}$ & 0,8 \\
\hline $\begin{array}{l}\text { Infiltration à partir des eaux de } \\
\text { crue }\end{array}$ & 1 & $\begin{array}{c}\begin{array}{c}\text { Evaporation de l'eau au } \\
\text { niveau des Foums. }\end{array} \\
\end{array}$ & 2,66 \\
\hline $\begin{array}{c}\text { Ré-infiltration des eaux } \\
\text { d'Irrigation }\end{array}$ & 0,34 & $\begin{array}{l}\text { Flux aval au niveau de la } \\
\text { limite Est de la nappe. }\end{array}$ & 0,18 \\
\hline Total & 10,23 & Total & 17,26 \\
\hline Bilan & & $-7,03$ & \\
\hline
\end{tabular}

\section{Conclusion}

La vulnérabilité de la nappe de la Feija est liée à ses caractéristiques géographiques et climatique, elle est également confirmé sa vulnérabilité en termes quantitatif (problèmes de surexploitation et prélèvements croissants) que qualitatif (augmentation de minéralisation et pollution). Tenant compte les entrés et les sorties actuelles de la nappe de la Feija, avec un réserve estimé de $104.3 \mathrm{Mm}^{3}$, on peut tracer d'une manière approximative la courbe prévisionnel de l'évolution de la réserve de la nappe, à partir de ce dernier la demande en eau en 2028 dépasserait la ressource disponible, et consiste à l'épuisement totale à l'horizon de 2031. Le bilan de la nappe de la Feija montre un déficit de $7.03 \mathrm{Mm}^{3}$, dont l'approche agronomique des besoins en eau des cultures résulte un volume des prélèvements agricole de l'ordre de $13.62 \mathrm{Mm}^{3}$, déjà ce volume est supérieur à l'ensemble des apports de la nappe. A cet effet il faut penser au problème de réserve et mettre l'accent sur l'amélioration de l'efficacité des modes de gestion dont la meilleur façon est celui d'un contrat de nappe qui permet la gestion de la nappe dans une approche participative et intègre l'ensemble des usagers dans la gestion des ressources en eau souterraines.

\section{Remerciements}

Nos remerciements s'adressent aux responsables de tous les services de l'Agence du Bassin Hydraulique Draa oued Noun pour leur aide fructueuse et la mise à notre disposition de tous les moyens logistiques, données et documents. 
Les auteurs tiennent à remercier également le GLOVIS-GATE de la NASA-USGS pour les données satellitaires (Landsat 8 Oli).nous sommes très reconnaissants aux évaluateurs anonymes pour leurs précieux commentaires et suggestions, ainsi qu'au rédacteur en chef de la revue pour son examen attentif de l'article, qui a permis de présenter ce travail.

\section{Références}

[1] Agence Du Bassin Hydraulique Souss Massa Draa (2013). Etude hydrogéologique de la nappe de la Feija, synthèse, élaboration des cartes hydrogéologique du système aquifère et proposition de points de dégagement.

[2] Algouti, Ab., Algouti, Ah., Chbani, B., Zaim, M. (2001). Sédimentation et volcanisme synsédimentaire de la série de l'Adoudounien infracambrien à travers deux exemples de I'Anti-Atlas du Maroc, Journal of African Earth Sciences, 32(4), 541-556.

[3] Boko, M., Niang, I., Nyong, A., Vogel, C., Githeko, A., Medany, M., Osman E.B., Tabo, R., Yanda, P. (2007). Africa climate change impacts, adaptation and vulnerability. In: Parry ML, Canziani OF, Palutikof JP, van der Linden PJ, Hanson CE (eds) Contribution of working group II to the fourth assessment report of the Intergovernmental Panel on Climate Change. Cambridge University Press, Cambridge, $433-467$.

[4] Buggish, W., \& Flügel, E. (1988). The Precambrian / Cambrian boundary in the Anti-Atlas (Morocco) discussion and new results, in The Atlas System of Morocco, V.H. Jacobshagen, Notes de lecture Earth Sci. Springer-Verlag, New York, 15, 361-404.

[5] Chamayou, J. (1966). Hydrogéologie de la vallée du Drà moyen. [Thèse de doctorat, Université de Montpellier].

[6] Cherkaoui, H.D., Moussadek, R., Sahbi, H. (2007). Apport des techniques géo-spatiales pour la caractérisation de la qualité des eaux souterraines des oasis de la vallée du Draa : cas de la nappe de Fezouata. (Options Méditerranéennes : Série B. Etudes et Recherches, 2(56), 295.

[7] Choubert, G. (1943). L'Acadien et l'Ordovicien de l'Anti- Atlas C.R. Acad. Sci. Paris, 216(3),158-160.

[8] Choubert, G. (1952). Histoire géologique du domaine de l'Anti-Atlas. 19e congr. Géol. Int. 3e Série, Maroc, 6, 77-194.

[9] Choubert, G. (1963). Histoire géologique du Précambrien de l'Anti-Atlas. Tome I. Notes et M. Sert. géol. Maroc, $162,352$.

[10] Choubert, G., \& Marçais J., (1952). Aperçu structural In: Géologie du Maroc Notes et Mém. Serv. Géol. Maroc, 100, fasc 1, partie 1 - et : 19ème Congr. Géol. Int, Alger 1952, Monogr région, 3ème série Maroc, 6, 9-73.

[11] Destombes, J. (1960). Stratigraphie de l'Ordovicien de la partie occidentale du jbel Bani et du jbel Zini, Anti-Atlas occidental (Maroc). B Soc. géol. Fr. 7ème série, 2(6), 747-751.

[12] El Mandour, A., El Yaouti, F., Fakir, Y., Zarhloule, Y., \& Benavente, J. (2007). Evolution of groundwater salinity in the unconfined aquifer of Bou-Areg, Northeastern Mediterranean coast, Morocco. Environmental Geology, 54(3), 491-503.

[13] Farah, A., Algouti, A., \& Algouti, A. (2021). Sedimentological and Lithostratigraphic Study of the Senonian Series in Timstiguite Region, Central High Atals, Morocco. Journal of Environmental and Agricultural Studies, 2(1), 01-11.

[14] Heidecke, C. (2009). Economic analysis of water use and management in the Middle Drâa valley in Morocco. [Doctoral dissertation Universität zu Bonn].

[15] Hübener, H., Schmidt, M., Sogalla, M., Kerschgens, M. (2005). Simulating evapotranspiration in a semi-arid environment. Theoretical and Applied Climatology 80, 153-167.

[16] Pouchin, T. (2001). Elaboration d'un observatoire paysager-Application à l'estuaire de Seine. [Thèse de doctorat, Université du Havre, UMR 6063 du CNRS].

[17] Robaux, A. (1952). Notions générale sur les phénomènes hydrologiques au Maroc notes et mémoire n97, hydrogéologie du Maroc. 